\title{
Actualizaciones en Inversión Pública: EI Invierte.pe a 2 años de su entrada en vigencia, aspectos que llevaron a las modificaciones introducidas por el Decreto Legislativo $1432^{(*)}$
}

\author{
Actualities in public investment: The current situation of "Invierte.pe" at two \\ years from it's released that caused that the Legislative Decree 1432 had \\ to suffer some modifications
}

\author{
Karla Mónica Gaviño Masías ${ }^{(*)}$ \\ Perú - Pontificia Universidad Católica del Perú
}

Resumen: A dos años de la entrada en vigencia del Sistema Nacional de Programación Multianual y Gestión de Inversiones, la experiencia práctica a través de su puesta en marcha trajo como correlato la necesidad de efectuar importantes modificaciones a su norma de creación. En el presente artículo revisaremos los aspectos que llevaron a dichas modificaciones y su abordaje en el nuevo Decreto Legislativo 1432, que modificó el Decreto Legislativo que crea el Sistema Nacional de Programación Multianual y Gestión de Inversiones. Si bien puede ser discutible el nivel de descentralización con que ya contaba el SNIP y su enfoque en los servicios a cargo del Estado antes que en la obra por sí misma, lo cierto es que SNIP sirvió de base para la construcción del Sistema Nacional de Programación Multianual y Gestión de Inversiones, el cual recoge muchos de los aciertos de dicho Sistema e intenta mejorar otros aspectos que quedaron pendientes.

Palabras Clave: Invierte.pe - Gestión de Inversiones - Decreto Legislativo 1432 Sistema Nacional de Programación Multianual - Modificaciones

\begin{abstract}
It's been two years since the National Multiyear Programming and Investment Management System came into force. During this time, the practical experience helped to identify different aspects of its creation rules that needed to be improved. This article reviews those aspects and its regulation, according to the modifications contemplated in the new Legislative Decree 1432. While the level of decentralization already in place by the SNIP and its focus on state-run services rather than the work itself may be debatable, the truth is that SNIP served as the basis for the construction of the National Multi-Year Programming System and Investment Gestion, which captures many of the successes of the System and attempts to improve other aspects that remained outstanding.
\end{abstract}

Keywords: Invierte.pe - Investment Management - Legislative Decree Nº 1432 - National Multiyear Programming and Investment Management System - Modifications

(*) Nota del Editor: este artículo fue recibido el 15 de setiembre de 2019 y su publicación fue aprobada el 25 de octubre de 2019 . Este artículo elaborado en marzo de 2019.

${ }^{(* *}$ Abogada por la Pontificia Universidad Católica del Perú, Magíster en Finanzas y Derecho Corporativo por la Universidad ESAN, con estudios internacionales de posgrado. Profesora de la Escuela de Gestión Pública de la Universidad del Pacífico. Miembro extraordinaria de la Asociación Civil lus et Veritas. Contacto: gm8019@hotmail.com 


\section{Introduc ción}

La Inversión Pública en el Perú se encuentra regulada en el marco de un Sistema desde la publicación de la Ley 27293 , Ley del Sistema Nacional de Inversión Pública (SNIP), en el año $2000^{(1)}$, siendo posteriormente recogido como un Sistema Administrativo con la publicación de la Ley 29158, Ley Orgánica del Poder Ejecutivo (en adelante, LOPE) en diciembre de $2007^{(2)}$. A partir de la publicación del Decreto Legislativo 1252, Decreto Legislativo que crea el Sistema Nacional de Programación Multianual y Gestión de Inversiones, publicado el 01 de diciembre de 2016, y que entró en vigencia el 24 de febrero de 2017, se produjo la derogación del Sistema Nacional de Inversión Pública.

En un trabajo previo hemos expuesto cómo la creación del Invierte.pe respondió a razones políticas y a la necesidad de estrechar la vinculación de la inversión pública con la programación multianual orientada al cierre de brechas de infraestructura y acceso a servicios a cargo del Estado (Carrasco y Gaviño, 2017). Al respecto, el tercer considerando del Decreto Legislativo 1252 justifica la dación de la norma señalando que "corresponde emitir una norma con rango de Ley que reorganice el Sistema Nacional de Inversión Pública, sustituyéndolo por un sistema descentralizado y de acceso a servicios a cargo del Estado, para la efectiva prestación de servicios y la provisión de la infraestructura necesaria para el desarrollo del país" (sic).

En ese sentido, consideramos que un análisis sobre los resultados de la aplicación práctica del Invierte.pe a 2 años de su entrada en vigencia, si bien en principio podría parecer anticipado, es posible que sea realizado a la luz de la experiencia acumulada en inversión pública en el Perú en estos 19 años. Asimismo, atendiendo a que en setiembre de 2018 se produjeron importantes modificaciones al Decreto Legislativo de creación del Invierte.pe, a través del Decreto Legislativo 1432, consideramos importante analizar según su Exposición de Motivos, las causales de estas modificaciones, muchas de las cuales se han realizado siguiendo recomendaciones de organismos internacionales como la Organización para la Cooperación y el Desarrollo Económicos (OCDE), atendiendo a la experiencia en inversión pública a nivel mundial.

Entre las primeras principales conclusiones que puede extraerse de la aplicación del Invierte.pe está sin duda el dato económico: conforme a las cifras mostradas por el Ministerio de Economía y Finanzas (en adelante, MEF), la inversión pública registró un crecimiento del $16.1 \%$ en el 2018 en términos nominales, llegando a un monto de ejecución ascendente a S/. 32, 988 millones al cierre de dicho año, cifra superior a los S/. 28,406 millones del 2017(3). Es decir, la ejecución de inversiones fue largamente superior en el 2018 frente al 2017, y esa tasa de crecimiento de un año para el otro no ha tenido precedentes.

Este crecimiento superó incluso la cifra esperada de $14 \%$ para el 2018 prevista por el MEF en el Marco Macroeconómico Multianual publicado en abril de 2018, siendo la primera vez en los últimos 4 años que se tiene una cifra de doble dígito, y constituye en términos absolutos el nivel de inversión pública más alto en la historia del Perú (inclusive, se llegó en algunos meses del año a niveles superiores al $35 \%$ ). Un dato importante a resaltar es el aporte de los Gobiernos Locales en dicha cifra, ya que ejecutaron S/. 15, 051 millones, mientras el Gobierno Nacional ejecutó S/. 11,677 millones; y los Gobiernos Regionales, S/. 6, 260 millones, conforme lo ha señalado el MEF (2019).

Ante números tan importantes, resulta interesante preguntarnos lo siguiente: ¿Entonces por qué se consideró necesario el ajuste al Decreto Legislativo que crea el Invierte.pe? y, ¿cómo se abordó dicha modificación a la norma de creación del Invierte.pe? En el presente artículo intentaremos responder dichas interrogantes de forma sucinta a la luz de las razones brindadas por la Exposición de Motivos del Decreto Legislativo 1432.

\section{Análisis}

Una primera afirmación práctica puede ser que al cierre del 2018 aún se continuaban ejecutando proyectos de inversión formulados y evaluados en el marco del SNIP; tan es así, que incluso la Única Disposición Complementaria Transitoria del nuevo Reglamento del Decreto Legislativo $1252^{(4)}$, referida a la aplicación de las disposiciones del Sistema Nacional de Inversión Pública,

(1) Publicada en el Diario Oficial "El Peruano" el 28 de junio de 2000

(2) El inciso 7 del artículo 46 de la Ley 9158, Ley Orgánica del Poder Ejecutivo, explícitamente recoge a la Inversión Pública, como parte de las materias reguladas como Sistemas Administrativos.

(3) Portal de Semana Económica, nota publicada el 02.01.2019 www.semanaeconomica.com.

(4) Publicado en el Diario Oficial "El Peruano" el 09 de diciembre de 2018. 
reconoce que a la fecha se sigue aplicando la preinversión del SNIP, toda vez que dispone lo siguiente:

\begin{abstract}
"Los proyectos y programas de inversión que se encuentren en la fase de preinversión aplicando los contenidos mínimos de los estudios de preinversión respectivos del Sistema Nacional de Inversión Pública, de acuerdo a lo previsto en el artículo 15 del Reglamento del Decreto Legislativo 1252, aprobado por Decreto Supremo $N^{\circ}$ 027-2017-EF, continúan aplicando dichos contenidos hasta la declaración de viabilidad correspondiente. Para las demás fases se aplica la normativa del Sistema Nacional de Programación Multianual y Gestión de Inversiones" (Ver referencia).
\end{abstract}

Efectivamente, consideramos que estas cifras recogen el resultado de inversiones públicas formuladas y evaluadas previamente a su ejecución, tanto en el marco del SNIP como del Invierte.pe, lo cual no deslegitima que reflejen el estado de la inversión pública al cierre del 2018, y que su ejecución se haya realizado según las reglas del Invierte.pe, conforme lo disponían las normas de transitoriedad entre un sistema y otro contempladas en el antiguo reglamento del Invierte.pe, que disponía su aplicación para la ejecución de los proyectos incluso cuando hubiesen sido formulados según las disposiciones del SNIP.

A esto debe sumarse que los resultados descritos se produjeron en un año con una agitada coyuntura política nacional, por lo cual resalta más aún el buen desenvolvimiento de la inversión pública y las cifras registradas al cierre del 2018. Es justamente en este contexto político que a mediados de año, mediante Ley 30823, Ley que delega en el Poder Ejecutivo la facultad de legislar en materia de gestión económica y competitividad, de integridad y lucha contra la corrupción, de prevención y protección de personas en situación de violencia y vulnerabilidad, y de modernización de la gestión del Estado, ${ }^{(5)}$ el Congreso de la República delegó en el Poder Ejecutivo la facultad para legislar en múltiples materias, incluyendo la modernización del Estado. De manera concreta, los literales a. 1 y a. 3 del numeral 5 del artículo 2 de esta ley incluyen la potestad para regular el fortalecimiento de la interoperabilidad, articulación e integración entre los sistemas conformantes de la Administración Financiera del Sector Público y sus respectivos órganos rectores, actualizar y adecuar sus conceptos y terminología, de manera que permita garantizar una gestión más integral de la Hacienda Pública; y adecuar el marco regulatorio a los cambios en materia de inversión pública, inversión público-privada y programación multianual del gasto.

En este marco se publicó en setiembre último el Decreto Legislativo $1432^{(6)}$, Decreto Legislativo que modifica el Decreto Legislativo 1252, Decreto Legislativo que crea el Sistema Nacional de Programación Multianual y Gestión de Inversiones y deroga la Ley 27293, Ley del Sistema Nacional de Inversión
Pública, cuya Exposición de Motivos señala que si bien los cambios introducidos por el Invierte.pe han sido importantes para promover el desarrollo coordinado de las inversiones en los tres niveles de Gobierno, conforme a la planificación estratégica y a la programación presupuestaria de mediano plazo, "resulta necesario [dictar] medidas que permitan fortalecer dicho Sistema Nacional en un contexto en el que es prioritario dar mayor impulso a las inversiones para cerrar las brechas de infraestructura y provisión de servicios y al mismo tiempo que la inversión responda a criterios de calidad y transparencia en su ejecución. Con ello se busca promover un mayor impacto social, transparencia y mejora continua de las inversiones que se realizan en los tres niveles de Gobierno".

Para lograr este objetivo, la Exposición de Motivos identificó tres aspectos principales que requerían de una modificación con rango de ley, que para efectos del presente análisis podemos resumir de la siguiente forma: (i) mejorar la orientación de la programación multianual de las inversiones; (ii) fortalecer la coordinación interinstitucional entre los tres niveles de gobierno; y (iii) contar con mecanismos de rendición de cuentas sobre el avance del cierre de brechas.

A continuación, analizaremos dichos aspectos, y su concreción en las modificaciones al Decreto Legislativo del Invierte.pe efectuado por el Decreto Legislativo 1432.

\subsection{Mejorar la orientación de la} programación multianual de las inversiones Uno de los principales aportes del Invierte. pe respecto del SNIP, es la Programación Multianual, que se encuentra regida por una serie de criterios articuladores, a fin de que cada Sector del Gobierno Nacional, Gobierno Regional o Gobierno Local pueda elaborar como resultado de este ejercicio su "Programa Multianual de Inversiones" (en adelante, PMI). Cabe señalar que los PMI son posteriormente consolidados por la Dirección General de Inversión Pública del MEF en su

(5) Publicada en el Diario Oficial "El Peruano" el 19 de julio de 2018.

(6) Publicado en el Diario Oficial "El Peruano" el 16 de setiembre de 2018. 
Actualizaciones en Inversión Pública: El Invierte.pe a 2 años de su entrada en vigencia, aspectos que llevaron a las modificaciones introducidas por el Decreto Legislativo 1432

Actualities in public investment: The current situation of "Invierte.pe" at two years from it's released that caused that the Legislative Decree 1432 had to suffer some modifications

calidad de rector de este Sistema, dando como producto el Programa Multianual de Inversiones del Estado (en adelante, PMIE).

El literal a) del artículo 3 del DLEG 1252 establece como un principio rector que el PMI se realiza considerando como principal objetivo el cierre de brechas de infraestructura y acceso a servicios públicos. Asimismo, a lo largo del texto del DLEG 1252, podemos encontrar vinculaciones expresas de la programación multianual con los objetivos previstos en los planes estratégicos de los distintos niveles de gobierno, y con sus recursos, con la finalidad de que estos se destinen a inversiones previstas en el PMI, en concordancia con la planificación estratégica del Sistema Nacional de Planeamiento Estratégico.

Si bien en teoría esta concordancia nos debería llevar a una mayor articulación entre el Planeamiento, la Inversión y el Presupuesto, lo cierto es que en la práctica gran número de inversiones para las cuales se requería financiamiento no se encontraban recogidas en el PMI y, por tanto, no necesariamente se había realizado el análisis de si coadyuvaban o no al cierre de brechas de infraestructura o acceso a servicios, o si se encontraban o no alineadas al planeamiento estratégico.

Así, la Exposición de Motivos del DLEG 1432 señala que, para el año 2018, en total 1,218 entidades públicas aprobaron $y$, posteriormente, presentaron al MEF su Programación Multianual de Inversiones (PMI), lo que representa al 95\% de Entidades Públicas que cumplieron con registrar en el Banco de Inversiones del Invierte.pe al órgano encargado de elaborar el PMI del Sector del Gobierno Nacional, Gobierno Regional - Gobierno Local, es decir, a la Oficina de Programación Multianual de Inversiones (OPMI). Esta cifra resulta bastante positiva, sin perjuicio de lo cual la Exposición de Motivos precisa que, tras la presentación de los PMI, las propias Entidades presentaron a las OPMI más de 2.817 solicitudes de inversiones no previstas para ser incorporadas en sus PMI (cada solicitud puede contener varias inversiones). Como resultado del análisis de estas solicitudes, se incorporaron más de 6, 883 inversiones, por un monto total mayor a S/. 4, 427 millones.

Lo expuesto no debe confundirse con el carácter dinámico propio de la Programación Multianual y del PMI, que es retroalimentado en cada fase del Ciclo de Inversión; por ejemplo, puede adelantarse de un año para otro la ejecución de proyectos previstos en el PMI conforme se consiguen mayores recursos para financiar más inversiones, o puede adelantarse o postergarse de un año para otro el avance de las inversiones previstas según sus cronogramas de ejecución sufran alteraciones como producto de procesos de selección o controversias arbitrales, entre otros supuestos. En estos ejemplos estamos siempre en el escenario de modificaciones al interior del PMI; es decir, de inversiones que ya han pasado el filtro del cierre de brechas como parte de la fase de Programación Multianual y todas las articulaciones que esta debe implicar.

Las cifras antes mencionadas evidenciaban dos situaciones, conforme lo describe la Exposición de Motivos:

a) La programación de las inversiones no se encontraba articulada con el financiamiento de las inversiones (es decir, el presupuesto), toda vez que muchas inversiones no previstas en el PMI habían sido consideradas en la Ley de Presupuesto del Año Fiscal 2018, en tanto que otras inversiones efectivamente consignadas en el PMI no contaban con presupuesto.

b) La práctica arrojaba escenarios en los cuales las necesidades de inversión podían surgir en diferentes momentos y variar en cada sector, según distintos acontecimientos o eventos no previstos. Ello ameritaba una programación multianual de las inversiones más flexible, manteniendo la vinculación con los objetivos priorizados, metas e indicadores que regulan esta programación.

Asimismo, la Exposición de Motivos también identificó la necesidad de incorporar el criterio de rendición de cuentas, entre los recursos ejecutados y las brechas por cerrar:

“(...) desde la entrada en vigencia del Sistema Nacional de Programación Multianual y Gestión de Inversiones se ha podido advertir que algunas normas del Decreto Legislativo $\mathrm{N}^{\circ} 1252$ han generado que los actores involucrados en el ciclo de las inversiones hayan realizado una programación multianual enfocada en la cartera de inversiones, concentrándose de esta manera casi únicamente en la identificación de una lista de proyectos a ejecutarse, perdiendo relevancia los objetivos, metas e indicadores establecidos en la programación multianual, los cuales deberían orientar las inversiones y a su vez permitir que se evalúe el avance en el cierre de brechas de infraestructura o de acceso de servicios públicos. Esta situación debilita la gestión estratégica de las inversiones y resta dinamismo a la ejecución del gasto".

Para hacer frente a esta situación, el Decreto Legislativo 1432 pone énfasis en la vinculación de objetivos y metas con la 
priorización y asignación de los recursos, fortaleciendo la base de la programación presupuestaria, a fin de que se considere a todas las inversiones que requieren recursos públicos, y que en la fase de ejecución presupuestaria, las modificaciones en materia de inversiones respondan al logro de objetivos estratégicos de las entidades públicas, con miras a poder evaluar al final de cada ejercicio el uso de los fondos públicos asignados contra el avance en el cierre de brechas; es decir, analizar como sector del Gobierno Nacional, como Gobierno Regional o como Gobierno Local cuánto gasté frente a cuánto de la brecha cerré. Respecto de la rendición de cuentas a la población, profundizaremos más adelante.

Para regular este aspecto, el artículo 1 del Decreto Legislativo bajo comentario modifica el literal b) y c) del artículo 3 del Decreto Legislativo 1252, estableciendo como principios rectores del Invierte.pe lo siguiente:

b) La programación multianual de la inversión vincula los objetivos nacionales, regionales y locales establecidos en el planeamiento estratégico en el marco del Sistema Nacional de Planeamiento Estratégico, con la priorización y asignación multianual de fondos públicos a realizarse en el proceso presupuestario, y debe realizarse en concordancia con las proyecciones del Marco Macroeconómico Multianual.

c) Los fondos públicos destinados a la inversión deben relacionarse con la efectiva prestación de servicios y la provisión de la infraestructura necesaria para el desarrollo del país, con un enfoque territorial.

Esta modificación no implica dejar atrás la elaboración de una cartera de proyectos a ejecutar, ni el análisis de las brechas de infraestructura o acceso a servicios públicos que se encontraba antes recogido en ambos literales, toda vez que estos elementos se encuentran recogidos a lo largo de las nuevas normas del Invierte.pe, sino que fortalece expresamente en la norma la vinculación con los otros sistemas administrativos (Planeamiento y Presupuesto), y la asignación de recursos públicos a favor de infraestructura y servicios de necesidad para el desarrollo territorial.

De la mano con este ajuste, se modifica toda la definición de la Fase de Programación Multianual de Inversiones, recogida en el literal a) del artículo 4 del Decreto Legislativo 1252, manteniendo la trianualidad del PMI y la búsqueda del cierre de brechas, e incorporando la evaluación del avance en el cierre de las mismas, a fin de realizar una debida rendición de cuentas (aspecto antes no previsto). Se va así directamente a la esencia del aspecto a mejorar conforme al diagnóstico de la Exposición de Motivos.

En ese sentido, el segundo párrafo de dicho nuevo literal dispone lo que a continuación citamos:

"Que dicha programación se elabora en función de los objetivos nacionales, regionales y locales establecidos en el planeamiento estratégico en el marco del Sistema de Planeamiento Estratégico, estableciendo metas para el logro de dichos objetivos que permitan evaluar el avance respecto al cierre de brechas de infraestructura o acceso a servicios y la rendición de cuentas. Constituye el marco de referencia orientador de la formulación presupuestaria anual de las inversiones. Incluye el financiamiento estimado para las inversiones a ser ejecutadas mediante el mecanismo de obras por impuestos, así como el cofinanciamiento estimado para los proyectos de inversión a ser ejecutados mediante asociaciones público-privadas cofinanciadas".

Otro aspecto importante para mantener actualizado el PMI es la modificación del numeral $5.3 \mathrm{del}$ artículo 5 del Decreto Legislativo 1252, introducida por el Decreto Legislativo 1432, referido a las funciones del órgano resolutivo, es decir, del ministro o máxima autoridad del sector, gobernador regional o alcalde, según el caso, precisando que tras la aprobación del PMI que realiza, solamente la modificación de los objetivos priorizados, metas e indicadores contenidos en el PMI deberá ser aprobado por este. Así, cualquier otra modificación al PMI, como la inclusión de nuevas inversiones en la cartera de inversiones, se debe efectuar conforme lo establezca el reglamento; es decir, por la OPMI, siempre que se enmarquen en lo antes expuesto.

Cabe señalar que el inciso 4 del numeral 9.3 del artículo 9 del Reglamento del Decreto Legislativo 1252 establece como función del órgano resolutivo (en adelante, OR) aprobar las brechas identificadas y los criterios de priorización de las inversiones a ser aplicadas en la elaboración de su PMI, de acuerdo a las medidas definidas por los Sectores, por lo que la norma permite que los cambios que no afecten las brechas y criterios de priorización aprobados por el OR sean aprobados por el órgano técnico que elabora el PMI, sin necesidad de llegar nuevamente al órgano político, en la lógica de que si los cambios se ciñen a lo aprobado por el OR, para qué volver a pasar nuevamente por este para cada cambio.

En ese sentido, no será posible incluir inversiones que no se alineen con estos criterios, cautelando así la orientación adecuada al cierre de brechas en la provisión 
Actualizaciones en Inversión Pública: El Invierte.pe a 2 años de su entrada en vigencia, aspectos que llevaron a las modificaciones introducidas por el Decreto Legislativo 1432

Actualities in public investment: The current situation of "Invierte.pe" at two years from it's released that caused that the Legislative Decree 1432 had to suffer some modifications

de infraestructura y acceso a servicios que persigue el Invierte. pe.

\subsection{Fortalecer la coordinación interinstitucional entre los tres niveles de gobierno}

Sobre el particular, la Exposición de Motivos señala la necesidad de procurar una mayor coordinación interinstitucional entre los distintos niveles de Gobierno. Consideramos que esta articulación es necesaria y traerá efectos positivos para contar con la identificación de los Indicadores de brechas (dados por los Sectores del Gobierno Nacional) que sirven de insumo a los tres niveles de Gobierno para la elaboración de su diagnóstico de brechas, así como para realizar inversiones de forma conjunta y/o articulada, evitando la duplicación de inversiones entre los distintos niveles de gobierno. Asimismo, conforme veremos en los siguientes párrafos, permitirá a los sectores del Gobierno Nacional revisar la correcta aplicación de sus metodologías de formulación y evaluación sectoriales, y de la calidad de las inversiones ejecutadas por los gobiernos regionales y gobiernos locales, en el marco de la descentralización y de sus competencias, pero que recaigan en la responsabilidad funcional del Sector.

Al respecto, los Principios de Acción para una inversión pública efectiva en todos los niveles de Gobierno, aprobados por la OCDE (2014), señalan lo siguiente:

“(...) la inversión pública efectiva entre los niveles de gobierno requiere de significativa coordinación para superar una serie de brechas que puedan existir en la información, en las políticas o en términos fiscales. La colaboración para las estrategias de inversión pública entre jurisdicciones y niveles del gobierno es difícil, incluso en situaciones en las que los actores involucrados claramente reconocen la necesidad de ella. Los costos de transacción, presiones competitivas, las limitaciones de recursos, prioridades diferentes y los temores de que la distribución de los costos o beneficios de la cooperación sea injusta, son ejemplos de elementos que pueden obstaculizar los esfuerzos de reunir a los gobiernos" (sic).

Estas preocupaciones se han recogido en las modificaciones materia de análisis. Así, el nuevo literal c) del artículo 3 del Decreto Legislativo 1252, modificado por el Decreto Legislativo 1432 antes citado, coloca en los principios del Invierte.pe el enfoque territorial de la programación. El segundo párrafo del nuevo numeral $5.3 \mathrm{del}$ artículo 5 establece que los OR de los sectores del Gobierno Nacional aprueban los indicadores de brechas y los criterios para la priorización de las inversiones realizadas en las funciones de su competencia, a ser aplicados en la fase de Programación Multianual de Inversiones por los tres niveles de gobierno. Con estos indicadores, los gobiernos regionales y gobiernos locales podrán identificar sus propias brechas.

El Decreto Legislativo 1432 introduce una modificación interesante al literal b) del numeral 4.1 del artículo 4 del
Decreto Legislativo 1252, señalando en su segundo párrafo que "en el caso de los Sectores, realizan, periódicamente, el seguimiento y evaluación de la calidad de las decisiones de inversiones de los tres niveles de gobierno, que se enmarquen en su ámbito de responsabilidad funcional" (sic).

Lo expuesto, consideramos, no debe interpretarse como una centralización discreta de la formulación y evaluación de los proyectos de los gobiernos regionales o gobiernos locales, toda vez que estos mantienen la facultad de formular y evaluar sus proyectos, siendo ellos mismos quienes los aprueban. La norma, al hacer referencia al seguimiento y evaluaciones periódicas sobre la calidad de las inversiones, busca justamente permitir que los sectores puedan participar, apoyar y fomentar la correcta aplicación de sus metodologías sectoriales, identificando aspectos a mejorar o aclarar para las futuras formulaciones y evaluaciones de proyectos por parte de los gobiernos regionales o gobiernos locales.

Adicionalmente, resulta de importancia resaltar lo dispuesto por la Tercera Disposición Complementaria Final del Decreto Legislativo 1252, introducida por el Decreto Legislativo 1432, referida a los criterios de priorización para el financiamiento de inversiones con transferencias, que establece que solo podrán recibir transferencias del Gobierno Nacional los gobiernos regionales y gobiernos locales cuyas inversiones hayan cumplido con los criterios aprobados por los sectores. La modificación introducida reemplaza la verificación que debía realizar el sector previamente a la transferencia, por el cumplimiento de los criterios de priorización dados por este.

En ese sentido, las modificaciones al Invierte.pe procuran orientar en distintas fases del Ciclo de Inversión a la interacción y articulación entre los distintos niveles de Gobierno.

\subsection{Contar con mecanismos de rendición de cuentas sobre el avance del cierre de brechas}

Uno de los principales aspectos del Ciclo de Inversión es su constante retroalimentación en cada una de sus 4 fases (fase de 
Programación Multianual de Inversiones, fase de Formulación y Evaluación, fase de Ejecución y fase de Funcionamiento). En ese sentido, iniciándose el ciclo con la identificación de las brechas en la provisión de infraestructura y acceso a servicios públicos, como parte de la Programación Multianual de Inversiones, resulta importante contar con un mecanismo de rendición de cuentas que permita identificar en qué medida las inversiones que realiza el Sector del Gobierno Nacional, gobierno regional o gobierno local se encuentran alineadas a dicha brecha o coadyuvan a su cierre, poniendo a disposición y en conocimiento de la población dicha información como parte de la transparencia que debe primar en la inversión pública y que, a su vez, permite relegitimar al Invierte.pe y a las entidades públicas ante la población. Este aspecto no se encontraba expreso en la regulación inicial del Invierte.pe, y mirando hacia atrás, probablemente sea la relegitimización de la inversión pública con la población el principal elemento que no logró concretar el antiguo SNIP y, consideramos, será a futuro uno de los más grandes retos que tenga el Invierte.pe, pues no basta con la exposición de datos económicos "duros" en la web, ni de mapas con información unidireccional como medida de datos abiertos que el SNIP intentó desarrollar, quizá el reto a futuro en materia de rendición de cuentas a la población incluya también el desarrollo de prácticas de gobierno abierto.

Al respecto, la Exposición de Motivos del Decreto Legislativo 1432, señala que "(...) resulta necesario que se cuente con un mecanismo de rendición de cuentas que permita informar a la población respecto al desempeño de los Sectores, Gobiernos Regionales y Gobiernos Locales en la gestión de la inversión pública con la finalidad de fomentar procesos transparentes en relación a los resultados alcanzados en términos de los servicios que recibe la población, lo cual permitirá la generación de confianza en la entidad" (sic).

Como se señaló previamente, el Decreto Legislativo 1432 modifica el Decreto Legislativo 1252 previendo como parte de la Programación Multianual de las Inversiones la rendición de cuentas a la población. Adicionalmente, el nuevo texto del artículo 11 introducido por el Decreto Legislativo 1432 hace énfasis en el seguimiento y evaluación de las inversiones, que tiene por objetos determinar el avance y cumplimiento del cierre de brechas de infraestructura y acceso a servicios de la cartera de inversiones; la ejecución y entrega de servicios a la población; y la retroalimentación para la mejora continua de la aplicación del Ciclo de Inversión y a la rendición de cuentas de los recursos públicos de inversión.

Para estos efectos, el nuevo texto del artículo 11 establece la remisión de información correspondiente a la ejecución presupuestaria a cargo del Sistema Nacional de Presupuesto Público; de la programación multianual de concertaciones y el plan anual de desembolsos de las operaciones de endeudamiento, a cargo del Sistema
Nacional de Tesoro Público; la información del gasto corriente ligado a la operación y mantenimiento de los activos creados con inversión pública del Sistema Integrado de Gestión Administrativa, a cargo del Sistema Nacional de Abastecimiento; y, el registro en el Banco de Inversiones, por parte de los órganos del Invierte.pe, de la evaluación de la calidad de las inversiones realizada por los sectores del Gobierno Nacional.

\section{Conclusiones}

Tras lo expuesto, podemos resumir las siguientes principales conclusiones con relación a las modificaciones del Decreto Legislativo 1252, introducidas por el Decreto Legislativo 1432:

a) Luego de 2 años de aplicación del Invierte. pe, ha sido posible identificar 3 aspectos relevantes susceptibles de mejora en su norma de creación, con la finalidad de mejorar la orientación de la programación multianual de las inversiones, de fortalecer la coordinación interinstitucional entre los tres niveles de gobierno y, de contar con mecanismos de rendición de cuentas sobre el avance del cierre de brechas. Para implementar estas mejoras, el Decreto Legislativo 1432 realizó distintos ajustes al Decreto Legislativo 1252, incluyendo la modificación prácticamente de toda la regulación del ciclo de inversiones, en lo referido a dichas materias.

b) La mejora en la orientación de la programación multianual de las inversiones ha sido abordada mediante la modificación de los principios que la rigen, así como de todo el artículo que la regula de forma específica. Las nuevas disposiciones para la Programación Multianual, mantienen la finalidad de lograr el cierre de brechas de infraestructura y servicios, mas ponen énfasis en la vinculación de objetivos y metas con la priorización, asignación y ejecución de los recursos, con miras a poder evaluar cuánto de lo gastado en inversión pública por parte de los sectores del Gobierno Nacional, los gobiernos regionales y los gobiernos locales ha 
permitido efectivamente avanzar en el cierre de brechas de infraestructura y servicios públicos. Asimismo, se incluyen facilidades para la actualización del Programa Multianual, siempre que no se alteren los objetivos priorizados, metas e indicadores contenidos en él.

c) A fin de fortalecer la coordinación interinstitucional entre los tres niveles de gobierno, las modificaciones al Invierte. pe procuran orientar en distintas fases del Ciclo de Inversión a la interacción y articulación entre los mismos. Esta articulación es necesaria y traerá efectos positivos para contar con la identificación de los Indicadores de Brechas (dados por los Sectores del Gobierno Nacional) que sirven de insumo a los tres niveles de Gobierno para la elaboración de su diagnóstico de brechas, así como para realizar inversiones de forma conjunta y/o articulada, evitando la duplicación de inversiones entre los distintos niveles de gobierno. Asimismo, permitirá a los Sectores del Gobierno Nacional revisar la correcta aplicación de sus metodologías de formulación y evaluación sectoriales, y de la calidad de las inversiones ejecutadas por los gobiernos regionales y gobiernos locales en el marco de la descentralización y de sus competencias, que recaigan en la responsabilidad funcional del Sector. d) Con relación a los mecanismos de rendición de cuentas sobre el avance del cierre de brechas, una de las novedades consiste en su mención en los principios del Invierte.pe y en el nuevo artículo 11 del Decreto Legislativo 1252, referido al seguimiento y evaluación de las inversiones. Si bien consideramos que su mayor regulación corresponderá a disposiciones de menor rango, así como al desarrollo de plataformas informáticas, resulta importante que desde las normas de mayor rango que regulan el Invierte.pe se incorpore esta visión, toda vez que la rendición de cuentas permita identificar en qué medida las inversiones que realiza el Sector del Gobierno Nacional, gobierno regional o gobierno local se encuentran alineadas al cierre de las brechas previamente identificadas, poniendo a disposición y en conocimiento de la población esta información como parte de la transparencia que debe primar en la inversión pública y que, a su vez, permite relegitimar al Invierte.pe y a las entidades públicas ante la población. Consideramos que este será a futuro probablemente uno de los mayores retos del Invierte.pe.

\section{Referencias bibliográficas}

Carrasco Carrasco, Gabriela y Gaviño Masías, Karla (2017). Aspectos Principales del Sistema Nacional de Programación Multianual y Gestión de Inversiones: Hacia un enfoque de inversiones programadas. Revista Administración Pública \& Control, 39, pág. 5-10.

Ministerio de Economía y Finanzas (03 de enero de 2019). Notas de Prensa. Ministerio de Economía y Finanzas. Recuperado el 3 de enero de 2019, de www.mef.gob.pe.

Organización para la Cooperación y el Desarrollo Económicos (OCDE) (2014). Principios de Acción para una inversión pública efectiva en todos los niveles de Gobierno. 